Homology, Homotopy and Applications, vol.4(2), 2002, pp.1-16

\title{
THE WORK OF JAN-ERIK ROOS ON THE COHOMOLOGY OF COMMUTATIVE RINGS
}

\author{
LUCHEZAR L. AVRAMOV \\ (communicated by Larry Lambe and Clas Löfwall)
}

Abstract

This is an attempt to present one aspect of the work of Jan-Erik Roos.

A glance at the list of publications reveals three clearly defined periods in his life as an algebraist. During the first one he studied abelian categories, obtaining fundamental results on derived functors of inverse limits. They are contained in $[\mathbf{3}]$, $[\mathbf{5}]-[\mathbf{9}],[\mathbf{1 1}]-[\mathbf{1 7}],[\mathbf{1 9}]-[\mathbf{2 1}]$. In the second period he focused on the homological theory of non-commutative rings, producing methods and results of lasting interest, among them a truly classic theorem - the determination of the global dimension of Weyl algebras. The papers $[\mathbf{4}],[\mathbf{1 8}],[\mathbf{2 2}]-[\mathbf{2 6}]$, and $[\mathbf{3 1}]$ (treating related questions from commutative ring theory) contain the results of that period. Björk $[\mathbf{B} \mathbf{j}]$ has given an overview in the context of contemporary and subsequent research.

The work discussed here starts in the mid-1970s, when Jan-Erik turned to homological problems on finitely generated modules over commutative noetherian local (or graded) rings. He has produced fascinating results on the structure of free resolutions of modules of infinite projective dimension, and has investigated deep and mysterious links between homological properties of commutative rings and topological spaces. His study of numerical invariants encoded in Poincaré series, and of algebraic invariants determined by Yoneda products and by homology products, brings an unusual degree of integration between these components.

This highly original and technically difficult work also brings to mind other qualities, such as elegance and optimism. A quick look at the many rings appearing on the following pages shows that there is nothing contrived about his 'examples': they are defined by the kind of simple expressions in few variables that one scribbles on a piece of paper to have something 'concrete' to play with. Appearances notwithstanding, some of these rings have been craftily constructed to posess a desired property. Others have been found by sifting, with the determination of a gold prospector, through computations of (literally!) thousands of examples. The purpose in this survey is to provide a guide to some of Jan-Erik's finds.

A different perspective of work completed by 1985 can be found in the article of Anick and Halperin $[\mathbf{A H}]$. Connection with topology, which were discussed early on by Lemaire $[\mathbf{L e}]$ in a Bourbaki talk, and recently by Hess $[\mathbf{H e}]$ in historical context,

L. L. A. was partly supported by a grant from the N.S.F.

Received May 17, 2002, revised May 21, 2002; published on July 12, 2002.

2000 Mathematics Subject Classification: 01-02, 13-03, 13D, 55-04.

Key words and phrases: cohomology, commutative rings, homotopy Lie algebra, Hilbert series.

(C) 2002, Luchezar L. Avramov. Permission to copy for private use granted. 
are not pursued very far here. Choices had to be made for a framework in which to present the algebraic results. For simplicity and uniformity, all of them are stated in the classical situation of commutative graded algebras over a field, generated by finitely many elements of degree 1 . Another decision concerns the presentation and referencing of results that motivated Jan-Erik's research, or were triggered by it. A minimalist approach was adopted, so theorems of other authors are described only in the text, and bibliographic information on their papers is reduced to the year publication.

Several mathematicians whose work is touched upon in this survey got their degrees from Jan-Erik. At the end of te paper one can find a list of all his graduate students. Invariably, they had been drawn into research at the lively algebra seminar that Jan-Erik ran for decades with contagious enthusiasm. It is there that many results discussed below were first reported, and numerous collaborations started.

No attempt to describe Jan-Erik's influence on the development of the subject would be complete without a reference to the meeting on Algebra, Algebraic Topology, and their Interactions that he organized in 1983 in Stockholm. The exchange of ideas between two groups of mathematicians, one working in commutative algebra and the other in homotopy theory, reached an incredible level of intensity. It can be felt even today from the volume of proceedings [33], which carried the impact of the meeting far beyond the already large circle of its participants.

\section{Background}

Due to the variety of concepts and techniques used in Roos's research, it is not easy to point to a standard text for prerequisites. One purpose of this introductory section is to provide students of commutative rings with basic information on the non-commutative algebra and homological algebra used in the theorems. Another is to introduce notation that will stay fixed for the rest of the paper.

\subsection{Hilbert series}

Let $k$ be a field and $B=\bigoplus_{j \in \mathbb{N}} B_{j}$ be a graded $k$-algebra, with $B_{0}=k$ and $\operatorname{rank}_{k}\left(B_{j}\right)<\infty$ for all $j \in \mathbb{N}$. Let $V=\bigoplus_{j \in \mathbb{N}} V_{j}$ be a graded left $B$-module. We write $V(s)$ for the graded $B$-module with $V(s)_{j}=V_{j+s}$ for all $j$. If $V$ is finitely generated, then $\operatorname{rank}_{k} V_{j}<\infty$ for all $i \in \mathbb{N}$, so it has a Hilbert series

$$
V(y)=\sum_{j \in \mathbb{N}} \operatorname{rank}_{k}\left(V_{j}\right) y^{j} \in \mathbb{Z}[[y]] .
$$

Hilbert series of spaces $V=\bigoplus_{j \in \mathbb{N}} V^{j}$ graded by upper degrees are defined similarly.

\subsection{Poincaré series}

The module $V$ has a graded resolution $\varepsilon: F \rightarrow V$ where $F_{i}$ a direct sum of graded modules $B(s)^{b_{i s}}$ with $b_{i s} \in \mathbb{N}$ for all $i, s$ and $b_{i s}=0$ for all $s<i$. For $k=B / B_{\geqslant 1}$ one obtains graded vector spaces $\operatorname{Tor}_{i}^{B}(V, k)=\bigoplus_{j \in \mathbb{N}} \operatorname{Tor}_{i}^{B}(V, k)_{j}$ for all $i \in \mathbb{N}$. The 
Poincaré series of $V$ over $B$ is the formal power series

$$
P_{V}^{B}(y, z)=\sum_{i \in \mathbb{N}}\left(\operatorname{Tor}_{i}^{B}(V, k)(y)\right) z^{i} \in \mathbb{Z}[[y, z]] ;
$$

Abusing notation, we let $k$ denote the graded $R$-module $R / R_{\geqslant 1}$, and set $P^{B}(y, z)=$ $P_{k}^{B}(y, z)$. Poincaré series determine Hilbert series via an equality

$$
P_{V}^{B}(y,-1)=\frac{V(y)}{B(y)}
$$

where the left hand side is the limit of the sequence $\left(\sum_{i=0}^{r}(-1)^{i} \operatorname{Tor}_{i}^{B}(V, k)(y)\right)_{r \in \mathbb{N}}$, which converges in the $(y)$-adic topology of $\mathbb{Z}[[y]]$.

If $B$ is left noetherian, then $V$ has a graded resolution $F$ as above by finitely generated free $B$-modules, so $\operatorname{Tor}_{i}^{B}(V, k)(y)$ is a polynomial for each $i$, that is,

$$
P_{V}^{B}(y, z) \in \mathbb{Z}[y][[z]] .
$$

In this case, one also has a Poincaré series in a single homological variable:

$$
P_{V}^{B}(z)=P_{V}^{B}(1, z) \text {. }
$$

\subsection{Yoneda products}

Pairings of Ext groups, defined by Yoneda, turn

$$
E(B)=\bigoplus_{i \in \mathbb{N}, j \in \mathbb{Z}} \operatorname{Ext}_{B}^{i}(k, k)^{j}
$$

into a bigraded algebra, the Yoneda algebra of $B$, and give $\operatorname{Ext}_{B}(V, k)$ (respectively, $\left.\operatorname{Ext}_{B}(k, V)\right)$ a structure of bigraded left (respectively, right) module over it.

A Hopf algebra is a graded algebra $B$ equipped with a homomorphism of graded $k$-algebras $\Delta: B \rightarrow B \otimes_{k} B$, called the diagonal. If $B$ is a Hopf algebra, then the Yoneda algebra $E(B)$ is graded-commutative for the cohomological degree, that is

$$
\alpha \beta=(-1)^{i p} \beta \alpha \quad \text { for all } \quad \alpha \in E^{i}(B)_{j} \quad \text { and all } \beta \in E^{q}(B)_{q}
$$

\subsection{Koszul duals}

For degree reasons $\bigoplus_{i \in \mathbb{N}} \operatorname{Ext}_{B}^{i}(k, k)^{i} \subseteq E(B)$ is a bigraded subalgebra. Regraded diagonally, that is, by assigning $\operatorname{Ext}_{B}^{i}(k, k)^{i}$ degree $i$, it is called the Koszul dual algebra $B^{!}$of $B$. Priddy (1970) proved that $B^{!}$is is generated in degree 1 and its ideal of relations is generated in degree 2 .

He also constructed a complex of free graded left $B$-modules

$$
K_{\bullet}(B)=\cdots \longrightarrow B \otimes_{k} B_{i}^{!}(-i) \stackrel{\partial_{i}}{\longrightarrow} B \otimes_{k} B_{i-1}^{!}(1-i) \longrightarrow \cdots \longrightarrow B \longrightarrow 0
$$

with $\mathrm{H}_{0}\left(K_{\bullet}(B)\right)=k$ and each $\partial_{i}$ determined by the product $B_{1}^{!} \otimes_{k} B_{i-1}^{!} \rightarrow B_{i}^{!}$. In case $B$ is generated by $B_{1}$, he proved that the following conditions are equivalent:

(3.1) The complex $K_{\bullet}(B)$ of $(2)$ is exact.

(3.2) $P^{B}(y, z)=B^{!}(y z)$

(3.3) $E(B)=B^{!}$ 
(3.4) $B^{! !} \cong B$

When these conditions are satisfied, the algebra $B$ is said to be Koszul.

\subsection{Commutative algebras}

Let $S=k\left[x_{1}, \ldots, x_{e}\right]$ be a polynomial ring on indeterminates of degree 1 , let $I$ be an ideal generated by forms of degree at least 2 , and set $R=S / I$. Let $M$ be a graded $R$-module, finitely generated in non-negative degrees. A famous theorem of Hilbert yields

$$
M(y)=\frac{p(y)}{(1-y)^{e}} \quad \text { for some } \quad p(y) \in \mathbb{Z}[y] .
$$

As $R$ is commutative, $\operatorname{Tor}^{R}(k, k)$ has a homology product that turns it into a bigraded algebra, graded-commutative for the homological degree.

\subsection{Homotopy Lie algebras}

The $k$-dual of each $\operatorname{Tor}_{i}^{R}(k, k)_{j}$ is naturally isomorphic to $\operatorname{Ext}_{R}^{i}(k, k)^{j}$, and the homology product dualizes to a diagonal map turning $E(R)$ into a bigraded Hopf algebra. Milnor and Moore (1965), for $\operatorname{char}(k)=0$, André (1971), for $\operatorname{char}(k)>2$, and Sjödin (1980), for $\operatorname{char}(k)=2$ proved that such a Hopf algebra is the universal enveloping algebra of a bigraded Lie algebra $\pi(R)$, called the homotopy Lie algebra of $R$. When $\operatorname{char}(k)=0$ it is described by

$$
\pi(R)=\{\xi \in E(R) \mid \Delta(\xi)=\xi \otimes 1+1 \otimes \xi\}
$$

Set $\varepsilon_{i j}(R)=\operatorname{rank}_{k} \pi^{i}(R)^{j}$ and call $\varepsilon_{i}(R)=\sum_{j \in \mathbb{Z}} \varepsilon_{i j}(R)$ the $i$ 'th deviation of $R$.

The Lie algebra $\pi(R)$ yields compact descriptions of the other invariants of $k$. By the Poincaré-Birkhoff-Witt Theorem, if $\left\{\xi_{u}\right\}_{u \in \mathbb{N}}$ is a homogeneous basis of $\pi(R)$, then $E(R)$ has a $k$-basis consisting of the distinct products $\xi_{u_{1}}^{n_{1}} \cdots \xi_{u_{s}}^{n_{s}}$ with $u_{1}<$ $\cdots<u_{s}, n_{r} \geqslant 0$ if $u_{r}$ has even homological degree $u_{r}$, and $n_{r}=0,1$ otherwise. The Lie bracket in $\pi(R)$ give the multiplication table of basis elements. The deviations determine the Poincaré series through the formula

$$
P^{R}(y, z)=\frac{\prod_{h, j=0}^{\infty}\left(1+y^{j} z^{2 h+1}\right)^{\varepsilon_{2 h+1, j}(R)}}{\prod_{h, j=0}^{\infty}\left(1-y^{j} z^{2 h+2}\right)^{\varepsilon_{2 h+2, j}(R)}}
$$

The Koszul dual $R^{!}$is the universal enveloping algebra of the Lie algebra $\pi^{(1)}(R)$, obtained by diagonally regrading the subalgebra $\bigoplus_{i \in \mathbb{N}} \pi^{i}(R)^{i}$ of $\pi(R)$. It is a quotient of the free associative $k$-algebra on indeterminates $\xi_{1}, \ldots, \xi_{e}$ of degree 1 by a two-sided generated by linear combinations of $\xi_{i} \xi_{j}+\xi_{j} \xi_{i}$ and $\xi_{i}^{2}$ for $1 \leqslant i \leqslant j \leqslant e$.

\subsection{Homology of loop spaces}

Let $X$ be a connected CW complex of finite type. The singular cohomology ring $\mathrm{H}^{*}(X ; k)$ with coefficients in $k$ is then a gradaded-commutative $k$-algebra with $\operatorname{rank}_{k} \mathrm{H}^{n}(X ; k)<\infty$ for each $n \geqslant 0$, and $\mathrm{H}^{n}(X ; k)=0$ for $n>\operatorname{dim} X$. We let $R_{X ; k}$

denote the commutative $k$-subalgebra of $\mathrm{H}^{*}(X ; k)$ generated by $\mathrm{H}^{2}(X ; k)$, graded by assigning degree $i$ to the elements of $\mathrm{H}^{2 i}(X ; k)$. 
If $\Omega X$ is the loop space of $X$, then the singular homology $\mathrm{H}_{*}(\Omega X ; k)$ is a graded vector space of finite rank in each degree. Furthermore, composition of loops endows it with a structure of a (non-commutative, in general) associative $k$-algebra.

\section{Irrational Poincaré series}

By Hilbert's Syzygy Theorem, over the polynomial ring $S=k\left[x_{1}, \ldots, x_{e}\right]$ for each finitely generated module $M$ one has $P_{M}^{S}(y, z) \in \mathbb{Z}[y, z]$. On the other hand, Serre's characterization of regularity shows that for $R=S / I$ with $0 \neq I \subseteq\left(x_{1}, \ldots, x_{e}\right)^{2}$, all non-negative powers of $z$ appear in the series $P^{R}(y, z)$. In the late 1950s Kostrikin and Shafarevich, Serre, and Kaplansky asked whether it always represents a rational function. In the same breath, Serre had also asked if the series $\mathrm{H}^{*}(\Omega X ; k)(z)$ is rational for every simply connected finite CW complex $X$ when $\operatorname{char}(k)=0$.

The simplest case of these questions are rings with $R_{i}=0$ for $i \geqslant 2$, and complexes with $\operatorname{dim} X \leqslant 2$, for which the easily computed answers

$$
P^{R}(y, z)=\frac{1}{R(-y z)} \quad \text { and } \quad \mathrm{H}^{*}(\Omega X ; k)(z)=\frac{z}{1+z-\mathrm{H}^{*}(X ; k)(z)}
$$

are roughly equivalent. Roos extended the equivalence a step further.

\subsection{Short algebras}

We say that the algebra $R$ is short if $R_{i}=0$ for $i \geqslant 3$. For such an algebra, Löfwall established in his thesis (1986) the relation

$$
\frac{1}{P^{R}(y, z)}=\frac{1}{R^{!}(y z)}-\frac{1}{z}\left(R(-y z)-\frac{1}{R^{!}(y z)}\right) .
$$

Roos gave a different proof of this result, and used it to link homological invariants of short algebras with those of short CW complexes, that is, simply connected finite CW complexes of dimension at most 4; clearly, if $X$ is short, then so is $R_{X ; k}$. Following Anick and Gulliksen, we say that two serie $P(z)$ and $Q(z)$ are rationally related if there exist polynomials $a(z), b(z), c(z), d(z) \in \mathbb{Z}[z]$, such that

$$
P(z)=\frac{a(z) Q(z)+b(z)}{c(z) Q(z)+d(z)} \quad \text { and } \quad a(z) d(z)-b(z) c(z) \neq 0 .
$$

Theorem ([28]). If $X$ is a short $C W$ complex and $\operatorname{char}(k)=0$, then the series $\mathrm{H}^{*}(\Omega X ; k)(z)$ and $P^{R_{X ; k}}(z)$ are (explicitly) rationally related. Furthermore, every short $\mathbb{Q}$-algebra is isomorphic to $R_{X ; \mathbb{Q}}$ for some short space $X$.

Short algebras and short CW complexes are very special objects in their respective categories. Roos' insight to focus on the properties of their series was validated by Anick and Gulliksen (1985), who proved that the Poincaré series of any object in one of these categories is rationally related to the Poincaré series of a short object.

\subsection{Artinian algebras}

Anick (1979) constructed a simply connected finite CW complex $X$ of dimension 4 for which the series $\mathrm{H}^{*}(\Omega X ; \mathbb{Q})(z)$ is transcedental. Roos's theorem in $\S 2.1$ then 
produced a short graded algebra $R$ for which $P^{R}(z)$ is transcendental. Soon after seeing Anick's result, Löfwall and Roos (1980) proved:

Theorem ([29]). If $R=k\left[x_{1}, x_{2}, x_{3}, x_{4}, x_{5}\right] / I$ where $I$ is the ideal

$$
\left(x_{1}^{2}, x_{2}^{2}, x_{3}^{2}, x_{5}^{2}, x_{1} x_{2}, x_{3} x_{5}, x_{1} x_{4}^{2}, x_{2} x_{4}^{2}, x_{3} x_{4}^{2}, x_{4}^{3}, x_{4}^{2} x_{5}, x_{1} x_{3}+x_{2} x_{4}+x_{4} x_{5}\right)
$$

then the Poincaré series $P^{R}(t)$ is transcendental.

Their approach differs from Anick's. Using classical cohomological techiques, they first construct a graded Lie algebra $L$, finitely generated in degree 1 and related in degree 2, whose universal enveloping algebra $A$ has transcendental Hilbert series $A(z)$. The commutative ring $R=A^{!} /\left(A^{!}\right) \geqslant 3$ is then short, and has $R^{!}=A$. Formula (6) then shows that $P^{R}(z)$ is transcendental. Roos [30] presents a highly readable account of this construction and of related developments, in particular of the proof by Bøgvad (1983) that the trivial extension of $R$ by its dualizing module is a Gorenstein artinian algebra with transcendental Poincaré series.

\subsection{Toric algebras}

The ring in the preceding theorem has a single non-monomial relation. It soon turned out that the presence of such a relation is unavoidable: Backelin (1982) proved that if $I$ is generated by monomials, then the series $P^{R}(y, z)$ is rational. In the 1990s, under the influence of toric geometry, homological properties of algebras with binomial relations came under close scrutiny. It was asked whether $P^{R}(y, z)$ is rational for all rings with binomial relations. Gasharov, Peeva, and Welker (2000) gave a positive answer for 'generic' defining binomials. However, Roos and Sturmfels showed that, in general, the answer is still negative:

Theorem ([43]). Let $k[u, v]$ be a polynomial ring with variables of degree 1 , and let $R$ be the graded $k$-algebra obtained from the graded subalgebra

$$
k\left[u^{36}, u^{33} v^{3}, u^{30} v^{6}, u^{28} v^{8}, u^{26} v^{10}, u^{25} v^{11}, u^{24} v^{12}, u^{18} v^{18}, v^{36}\right] \subset k[u, v]
$$

by dividing all degrees by 36. The Poincaré series $P^{R}(t)$ is then transcendental.

The series is computed through several reductions, using Levin's theories of Golod homomorphisms (1975) and of large homomorphisms (1985). From the theorem Fröberg and Roos [45] extracted an affine monomial curve with transcendental Poincaré series: the subalgebra $k\left[x^{18}, x^{24}, x^{25}, x^{26}, x^{28}, x^{30}, x^{33}\right]$ of $k[x]$.

\section{Yoneda algebras}

Since the Yoneda algebra $E(R)=\operatorname{Ext}_{R}(k, k)$ and the homotopy Lie algebra $\pi(R)$ determine each other, in the following discussion we freely swith between them.

Some of the deepest cohomological information on $R$ concerns the structure of the Lie algebra $\pi(R)$, and the behavior of the deviations $\varepsilon_{i}(R)=\operatorname{rank}_{k} \pi^{i}(R)$, cf. $\S 1.6$. In [32] Roos surveyed the subject until around 1980. To put his own contributions in context we sketch some additional results.

The algebra $R$ is said to be complete intersection if the ideal $I$ can be generated by a regular sequence. For such rings $\pi(R)$ is completely understood, and is small: 
Tate (1957) proved that $\pi^{i}(R)=0$ for all $i \geqslant 3$. On the other hand, if $R$ is not complete intersection, then Halperin (1987) proved that $\pi^{i}(R) \neq 0$ for all $i \geqslant 1$. Furthermore, drawing on a result of Félix, Halperin, and Thomas (1982) on the growth of the rational homotopy groups of Avramov (1984) showed that there is an infinite sequence of indices $0<s_{1}<\cdots<s_{j}<\cdots$ with bounded ratios $s_{j+1} / s_{j}$ and a real number $\gamma>1$ such that $\varepsilon_{s_{j}}(R)>\gamma^{s_{j}}$ for all $j \geqslant 1$.

\subsection{Generation}

Levin (1974) conjectured that $E(R)$ is always finitely generated as an associative $k$-algebra. This is equivalent to the finite generation of $\pi(R)$ as a Lie algebra. Löfwall (1986) proved that if $R$ is short in the sense of $\S 2.1$, then $\pi(R)$ is a semi direct product $\pi^{(1)}(R) \ltimes L(W)$, where $L(W)$ is the free Lie algebra on the graded vector space $V(1)$, where $V$ is the third syzygy in a minimal free resolution of the graded

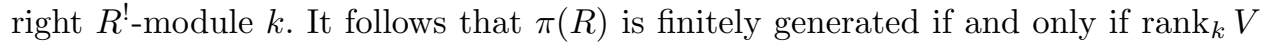
is finite. Here is a short ring for which this fails:

Theorem ([28]). If $R=k\left[x_{1}, x_{2}, x_{3}, x_{4}, x_{5}\right] / I$ where $I$ is the ideal

$$
\left(x_{1}^{2}, x_{2}^{2}, x_{3}^{2}, x_{4}^{2}, x_{5}^{2}, x_{2} x_{3}, x_{4} x_{5}, x_{1} x_{2} x_{4}, x_{1} x_{2}+x_{1} x_{3}+x_{1} x_{4}+x_{1} x_{5}\right)
$$

then the Yoneda Ext algebra $E(R)$ is not finitely generated.

The ring above was found by following the link to rational homotopy theory. Indeed, Halperin and Stasheff (1979), and others, had shown that short CW complexes are formal spaces. This has as a consequence an isomorphism of $\mathbb{Q}$-algebras $\mathrm{H}_{*}(\Omega X ; \mathbb{Q}) \cong E^{\prime}\left(\mathrm{H}_{*}(X ; \mathbb{Q})\right)$, where the prime indicates a regrading of the bigraded Ext algebra. The algebra $E\left(R_{X ; \mathbb{Q}}\right)$ is a retract of $E^{\prime}\left(\mathrm{H}_{*}(X ; \mathbb{Q})\right)$, and is equal to it in degree 1 . For $k=\mathbb{Q}$, the ring in the theorem is $R=R_{X ; \mathbb{Q}}$ where $X$ is a short CW complex, constructed by Lemaire (1974), with the property that the subalgebra of $\mathrm{H}_{*}(\Omega X ; \mathbb{Q})$ generated by $\mathrm{H}_{1}(\Omega X ; \mathbb{Q})$ is not finitely generated over $\mathbb{Q}$.

\subsection{Double Yoneda algebras}

For a graded $R$-module $M$, let $E(M)$ denote the bigraded $E(R)$-module $\operatorname{Ext}_{R}(M, k)$. By definition one has $E(R)(y, z)=P_{M}^{R}(y, z)$. Furthermore, $E^{2}(M)=$ $\operatorname{Ext}_{E(R)}(E(M), k)$ is a trigraded module over the trigraded algebra $E^{2}(R)$, and formula (1) yields $P_{M}^{R}(y, z)=1 / E(R)(y, z,-1)$. In particular, if $E(R)(y, z, u)$ is rational, then so is be $P_{M}^{R}(y, z)$. Now the algebra $E(R)$ is a Hopf algebra, cf. $\S 1.6$, hence the algebra $E^{2}(R)$ is graded commutative, cf. $\S 1.3$, and thus formula (4) would yield the desired rationality provided $E^{2}(R)$ is finitely generated as an algebra over $k$ and $E^{2}(M)$ is finitely generated as a module over it. Few would have even attempted such a long shot. Roos pulled it off twice.

A class of rings introduced by Golod (1962) has long served as testing ground for homological conjectures. Avramov (1974) and Löfwall (1986) independently showed that for a Golod ring $R$ the Lie algebra $\pi^{\geqslant 2}(R)$ is finitely generated and free. Using this fact, Roos proved the following

Theorem ([27]). If $R$ is Golod and $M$ is a finitely generated $R$-module, then the algebra $E(R)$ is coherent, the $E(R)$-module $E(M)$ is coherent, the commutative 
algebra $E^{2}(R)$ is finitely generated, the $E^{2}(R)$-module $E^{2}(R)$ is finitely generated, and the series $P_{M}^{R}(y, z)$ is rational with the same denominator as $P^{R}(y, z)$.

The last assertion had been proved earlier, by completely different means, by Ghione and Gulliksen (1975). In collaboration with Backelin, Roos undertook a systematic exploration of double Ext algebras. They applied it to prove the next

Theorem ([34]). If the ideal $I$ is generated by monomials in the indeterminates, then the commutative $k$-algebra $E^{2}(R)$ is finitely generated.

This is a far reaching generalization of Backelin's result that $P^{R}(y, z)$ is rational.

\subsection{Lie subalgebras generated in degree 1}

The Lie subalgebra $\pi^{(1)}(R)$ generated by $\pi^{1}(R)$, and the series of subdeviations $\delta_{i}(R)=\operatorname{rank}_{k} \pi^{(1)}(R)^{i}$, capture the impact of the quadratic relations of $R$. The extremal cases are easily described: At one end, $\delta_{2}(R)=0$ if and only if $\delta_{i}(R)=0$ for all $i \geqslant 2$, if and only if $R$ has no quadratic relation. At the other, $\delta_{i}(R)=\varepsilon_{i}(R)$ for all $i \geqslant 2$ if and only if $R$ is Koszul. In both cases, the Hilbert series $R^{!}(y)$ of the Koszul dual $R^{!}$, which is the universal enveloping algebra of $\pi^{1}(R)$, is rational. By a classical result of Kronecker, its denominator converges in the unit circle if and only if it is a product of cyclotomic polynomials, which here means $\delta_{i}(R)=0$ for all $i \gg 0$.

Non-complete intersection algebras $R$ with $\pi^{(1)}(R)$ is nilpotent can be described by the condition that the number $s(R)=\sup \left\{i \in \mathbb{N} \mid \delta_{i}(R) \neq 0\right\}$ is finite and at least 3 . Initially such rings were not easy to come by. The first examples were constructed by Löfwall around 1974 (unpublished). Kustin and Slattery (1994) found algebras with $s(R)=3$ having 4 generators and 5 relations. Hreinsdottir (1998), (2000) proved that for $n \geqslant 3$ the coordinate ring of the variety of 2 (respectivery, 3) commuting $n \times n$ matrices has $s(R)=3$ (respectively, $s(R)=4$ ). A systematic procedure described by Roos $[\mathbf{3 7}]$ shows that $s(R)$ can be any integer, and that the homological properties of rings with $s(R)<\infty$ deserve further study.

The question whether there exist rings with $\pi^{(1)}(R)$ non-nilpotent and with bounded subdeviations, was raised in the early eighties by Anick and Löfwall. An amazing positive answer was obtained by Löfwall and Roos.

Theorem ([42]). If $\operatorname{char}(k)=0$ and $R=k\left[x_{1}, x_{2}, x_{3}, x_{4}, x_{5}\right] / I$ where $I$ is the ideal

$$
\left(x_{2}^{2}, x_{2} x_{3}+x_{1} x_{4}, x_{3}^{2}-x_{2} x_{4}-x_{1} x_{5}, x_{3} x_{4}+x_{2} x_{5}, x_{4}^{2}\right)
$$

then for $i \geqslant 5$ the sequence of subdeviations of $R$ is periodic of period 4 , namely by

$$
\left(\delta_{i}(R)\right)_{i \geqslant 1}=(5,5,3,3, \underbrace{5,6,3,3}, \underbrace{5,6,3,3}, \underbrace{5,6,3,3}, \ldots)
$$

The construction of the algebra in the theorem is of considerable intrinsic interest, as it links up with Kac's (1977) classification of simple Lie superalgebras. A precedent had been set by Anick (1983), who produced a CW complex $X$ with cells in dimension 2 and 5 with rational homotopy Lie algebra $\pi(X) \otimes_{\mathbb{Z}} \mathbb{Q}$ exhibiting a similar periodic behavior. However, Anick's approach cannot be modified to yield algebras with relations in degree two, needed for the Koszul dual $R^{!}$. Löfwall and Roos use their methods to prove the following theorem. 
Theorem ([42]). There exists a CW complex $X$ with five 2-cells and seven 4 -cells. such thet $\mathrm{H}_{*}(\Omega X ; \mathbb{Z})$ has elements of order $p$ for every prime number $p$.

This space has many fewer cells then the original complexes with loop space homology torsion of every prime order, that Anick (1986) and Avramov (1986), obtained from an algebraic construction of Gulliksen, Fröberg, and Löfwall (1986).

\section{Koszul algebras}

Interest in Koszul agebras, defined in $\S 1.4$, has been spurred as much by their remarkable algebraic rigidity as by the unusual frequency with which they appear in solutions to completely different problems arising in algebraic topology, algebraic geometry, commutative algebra, representation theory, and combinatorics.

Koszul algebras have been steadily moving to the center of Roos' work.

\subsection{Recognition}

It has long been known that it is very difficult to determine whether a given algebra is Koszul. Eisenbud and Peeva asked if such a coclusion could be reached from knowing that the residue field $k$ has a resolution that is linear up to a certain possibly big, but a priori known degree. In response to that question, Roos proved the following remarkable result.

Theorem ([36]). Let $n \geqslant 2$ be an integer, and assume that $k$ has characteristic 0 . The $k$-algebra $R^{(n)}=k\left[x_{1}, x_{2}, x_{3}, x_{4}, x_{5}, x_{6}\right] / I^{(n)}$, where $I^{(n)}$ is the ideal

$$
\begin{gathered}
\left(x_{1}^{2}, x_{1} x_{2}, x_{2} x_{3}, x_{3}^{2}, x_{3} x_{4}, x_{4}^{2}, x_{4} x_{5}, x_{5} x_{6}, x_{6}^{2}\right. \\
\left.x_{1} x_{3}+n x_{3} x_{6}-, x_{4} x_{6}, x_{3} x_{6}+x_{1} x_{4}+(n-2) x_{4} x_{6}\right),
\end{gathered}
$$

have the same Hilbert series, but the Poincaré series are given by the formula

$$
\frac{1}{P^{R^{(n)}}(y, z)}=\frac{(1+y z)^{2}}{1-4 y z-(y z)^{2}+6(y z)^{3}+3(y z)^{4}}+\frac{(y z)^{n+1}(y+y z)}{(1+y z)^{2}} .
$$

In particular, the minimal resolution of the residue field of $R^{(n)}$ is linear for the first $n$ steps, but a non-linear term appears at the $(n+1)$ st step.

\subsection{Generalizations}

The starting point is the definition of Koszul algebras by means of condition (3.1). By dualizing over $k$ the differentials of the complex $K_{\bullet}(R)$ in (2), one obtains a complex of graded $R^{!}$-modules of the form

$$
K^{\bullet}(R)=\cdots \longrightarrow R_{i}^{*}(-i) \otimes_{k} R^{!} \longrightarrow R_{i-1}^{*}(1-i) \otimes_{k} R^{!} \longrightarrow \cdots \longrightarrow R^{!} \longrightarrow 0
$$

with $\mathrm{H}_{0}\left(K^{\bullet}(R)\right)=k$. Roos says that $R$ satisfies condition $\mathcal{L}_{t}$ for some integer $t \geqslant 2$ if $\mathrm{H}_{i}\left(K^{\bullet}(R)\right)=0$ for $i \neq 0, t-1$; note that condition $\mathcal{L}_{2}$ is equivalent to (3.4), hence to the Koszul property. Partly in collaboration with Löfwall, he proves:

Theorem ([37]). If $R$ satisfies condition $\mathcal{L}_{t}$ for some $t \geqslant 2$, then

$$
\frac{1}{P^{R}(y, z)}=\frac{1}{R^{!}(y z)}+\frac{1}{(-z)^{t-2}}\left(R(-y z)-\frac{1}{R^{!}(y z)}\right)
$$


For $t=3$ the formula above reduces to the expression (6) for the Poincaré series of a short algebras, as it should, since short algebras clearly satisfy condition $\mathcal{L}_{3}$. Further examples of generalized Koszul algebras are discussed in the next section.

\subsection{Algebras with quadratic relations}

When the number $e$ of generators of the graded $k$-algebra $R$ is small, its homotopy Lie algebra and its Poincaré series belong to a few well understood families: for $e \leqslant 2$ this due to Scheja (1964), and for $e=3$ to Avramov, Kustin, and Miller (1988) and to Weyman (1989).

For a number of year, Roos has been studying the quotients of $k\left[x_{1}, x_{2}, x_{3}, x_{4}\right]$ modulo ideals $I$ generated by quadratic forms. The ultimate goal of his investigation is a complete classification of all possible Hilbert series $R(y)$ and $R^{!}(y)$, and Poincaré series $P^{R}(y, z)$. So far, the most detailed account of his results is published in the form of tables in [40]. It contains a list of 60 algebras $R$ yielding all possible values of $R(y)$, and a possibly incomplete list of 83 algebras providing different values of $P^{R}(y, z)$. Surprisingly, 82 of these algebras satisfy $\mathcal{L}_{3}$, and the remaining one $\mathcal{L}_{4}$.

In parallel to the investigation of quadratic forms in 4 variables, Roos has been looking at ideals generated by quadrics in $k\left[x_{1}, x_{2}, x_{3}, x_{4}, x_{5}\right]$. The situation here is known to be more complicated: the theorems in $\S 3.1$ and $\S 2.2$ show that the Yoneda algebra may be infinitely generated, and that the Poincaré series may be transcendental. Unpublished results on the case $e=5$ spread over an intimidating number of cases (2500 at one count, and growing). They have been lovingly collected by Jan-Erik in several thick notebooks, which somehow appear to be always at hand. While it is difficult to predict whether this monumental effort will eventually lead to a complete classification, it has already produced results: some algebras encountered during the census campaign have raised suspicions - confirmed later - that they may have unusual properties, cf. $\S \S 2.3,3.3,4.4$.

Poincaré series in such quantities have been determined with extensive computer calculations based on the software packages MACAULAY of Bayer and Stillman, BERGMAN of Backelin, and CBAS of Löfwall and Pettersson. The mathematical and computational background underlying many computations is developed in [37]. More about the ideology and motivation behind his approach can be found in [39].

\subsection{Functional equation}

If $R$ is Koszul, then it satisfies an equality

$$
R(y) R^{!}(-y)=1
$$

obtained by eliminating theeseries $P^{R}(y,-1)$ from formulas (3.2). In his 1983 thesis, Backelin asked whether such an equality characterizes Koszul algebras. In 1985 he and Fröberg proved that this is indeed the case when $e \leqslant 3$. The theorem in $\S 4.2$ shows that if $R$ satisfies condition $\mathcal{L}_{t}$ for some $t \geqslant 2$, then equality (7) implies that $R$ is Koszul. Among the more that 2500 cases of algebras with quadratic relations in at most 5 variables with known homological invariants described in $\S 4.3$, only 4 non-Koszul algebras satisfy (7), cf. [41]. In particular, Roos proves: 
Theorem ([38]). If $R=k\left[x_{1}, x_{2}, x_{3}, x_{4}, x_{5}\right] / I$ where $I$ is the ideal

$$
\left(x_{1}^{2}+x_{1} x_{2}, x_{1}^{2}+x_{2} x_{3}, x_{1} x_{3}, x_{3}^{2}, x_{3} x_{4}+x_{2} x_{5}, x_{3} x_{5}, x_{4} x_{5}+x_{5}^{2}\right)
$$

then $R$ satisfies equation (7), but is not Koszul, and the following equality holds:

$$
\frac{1}{P^{R}(y, z)}=\frac{1}{R^{!}(y z)}+\frac{y^{5} z^{3}(1+x)}{(1+y z)^{2}} .
$$

Independently, Positselski also answered Backelin's question in 1995.

\subsection{Modules}

Let $R$ be a Koszul algebra. Formulas (1) and (4) show that the Poincaré series of its residue field is a rational function. Experience and mathematics both show that among all finitely generated $R$-modules $M$, the residue field $k$ is the one with the most complicated homological behavior, so there might be an understandable inclination to expect that every series $P_{M}^{R}(y, z)$ is rational. This is easily seen to be the case for $R=k\left[x_{1}, \ldots, x_{e}\right] /\left(x_{1}, \ldots, x_{e}\right)^{2}$, for every $e \geqslant 1$. Surprisingly, Jacobsson (1985) showed that for every $n \geqslant 3$ the Koszul algebra

$$
R^{[n]}=\frac{k\left[x_{1}, \ldots, x_{n}\right]}{\left(x_{1}, \ldots, x_{n}\right)^{2}} \otimes_{k} \frac{k\left[y_{1}, \ldots, y_{n}\right]}{\left(y_{4}, \ldots, y_{n}\right)^{2}}
$$

has modules with transcendental Poincaré series. Thus, two algebras defined by quadratic monomial relations exhibit fundamentally different homological behavior.

Roos' latest research has focused on the question of why such phenomena occur. He says that $R$ is good if there exists a polynomial $d_{R}(z)$, such that $d_{R}(z) P^{R}(z)$ is in $\mathbb{Z}[z]$ for all finitely generated $R$-modules $M$. In [46] he produces some sufficient conditions for $R$ to be good, and uses them to give examples of such algebras. However, he is mostly interested in bad Koszul algebras, and his main result is to show that the algebras $R^{[n]}$ above are as bad as they come.

Theorem ([46]). For every algebra $R$ there exists a graded module $M$ over some algebra $R^{[n]}$, such that the Poincaré series $P^{R}(z)$ and $P_{M}^{R^{[n]}}(z)$ are rationally related.

The construction of the module $M$ above is based on a careful analysis and further extensions of Jacobsson's construction. Jan-Erik would have been acting out of character, had he not taken the new toy apart and reassembled it in a different form - just to understand how it really works. Here is a result of this activity.

Theorem ([48]). Over the ring $R=\mathbb{C}\left[x_{1}, x_{2}, x_{3}, x_{4}\right] /\left(x_{1}^{2}, x_{1} x_{2}, x_{3} x_{4}, x_{4}^{2}\right)$, for each integer $p$ let $M_{p}$ be the module with presentation matrix

$$
\left(\begin{array}{ccc}
x_{2} & x_{1} & x_{4} \\
x_{3} & -x_{4} & x_{1}+2 \cos \left(\frac{2 \pi}{p}\right) x_{4}
\end{array}\right)
$$

When p ranges over the prime numbers, the Poincaré series $P_{M_{p}}^{R}(z)$ are all rational, and the radii of convergence of the series $\sum_{i=0}^{\infty} \operatorname{rank}_{k} \operatorname{Tor}_{i}^{R}(k, k)_{i} z^{i}$ converge to 1 .

This is related to a question of Avramov (1992). 


\section{Surveys related to work of Jan-Erik Roos}

[Le] Lemaire, Jean-Michel, Anneaux locaux et espaces de lacets à séries de Poincaré irrationnelles (d'après Anick, Roos, etc...), Séminaire Bourbaki (1980/81), Lecture Notes in Math. 901, Springer, Berlin-New York, 1981; pp. 149-156.

[AH] Anick, David J.; Halperin, Stephen, Commutative rings, algebraic topology, graded Lie algebras, and the work of Jan-Erik Roos, J. Pure Appl. Algebra 38 (1985), 103-109.

[Bj] Björk, Jan-Erik, Non-commutative noetherian rings and the use of homological agebra, J. Pure Appl. Algebra 38 (1985), 111-119.

[He] Hess, Kathryn, A history of rational homotopy theory, in: History of topology, North-Holland, Amsterdam, 1999; pp. 757-796.

\section{Theses directed by Jan-Erik Roos}

[1973] Ingegerd Palmér

[1976] Gunnar Sjödin

[1976] Clas Löfwall

[1976] Peter Strömbeck

[1983] Jörgen Backelin

[1983] Rikard Bøgvad

[1983] Hans Åberg

[1989] Erik Valtonen

[1991] Susanna Scrivanti (licentiate)

[1995] Mohammad Parhizgar (Clas Löfwall and Larry Lambe also played a role)

[1997] Freyja Hreinsdottir (Ralf Fröberg, Jörgen Backelin, and Clas Löfwall also played a role)

[1997] Emil Sköldberg (also directed by Ralf Fröberg) 


\section{Publications of Jan-Erik Roos}

[1] Roos, Jan-Erik, On subnormal differential operators in one variable with weak regularity requirements on the coefficients, ms. Lund, 1957; $22 \mathrm{pp}$.

[2] Gask, Hector; Roos, Jan-Erik, A simple model from potential theory, (Swedish) Nordisk Mat. Tidskr. 6 (1958), 5-20,

[3] Roos, Jan-Erik, Sur les foncteurs dérivés de lim. Applications, C. R. Acad. Sci. Paris 252 (1961), 3702-3704.

[4] Roos, Jan-Erik, Bidualité et structure des foncteurs dérivés de lim dans la catégorie des modules sur un anneau régulier, C. R. Acad. Sci. Paris $\mathbf{2 5 4}$ (1962), 1556-1558.

[5] Roos, Jan-Erik, Derived functors of infinite products and projective objects in abelian categories, 1962, ms. 16 pages (summary published in [11]).

[6] Roos, Jan-Erik, Introduction à l'étude de la distributivité des foncteurs $\varliminf_{\longleftarrow}$ par rapport aux $\mathrm{lim}_{\mathrm{W}}$ dans les catégories des faisceaux (topos), C. R. Acad. Sci. Paris 259 (1964), 969-972.

[7] Roos, Jan-Erik, Sur la distributivité des foncteurs $\lim _{\text {par rapport aux } \mathrm{lim}}$ dans les catégories des faisceaux (topos), C. R. Acad. Sci. Paris 259 (1964), $1605-1608$.

[8] Roos, Jan-Erik, Complément à l'étude de la distributivité des foncteurs lim par rapport aux lim dans les catégories des faisceaux (topos), C. R. Acad. Sci. Paris 259 (1964), 1801-1804.

[9] Roos, Jan-Erik, Caractérisation des catégories qui sont quotients de catégories de modules par des sous-catégories bilocalisantes, C. R. Acad. Sci. Paris 261 (1965), 4954-4957.

[10] Roos, Jan-Erik, An algebraic study of group and nongroup error-correcting codes, Information and Control 8 (1965), 195-214.

[11] Roos, Jan-Erik, Sur les foncteurs dérivés des produits infinis dans les catégories de Grothendieck. Exemples et contre-exemples, C. R. Acad. Sci. Paris Sér. A-B 263 (1966), A895-A898.

[12] Roos, Jan-Erik, Sur la condition $\mathrm{AB} 6$ et ses variantes dans les catégories abéliennes, C. R. Acad. Sci. Paris Sér. A-B 264 (1967), A991-A994.

[13] Roos, Jan-Erik, Locally distributive spectral categories and strongly regular rings, in: Reports of the Midwest Category Seminar, Springer, Berlin, 1967; pp. $156-181$

[14] Roos, Jan-Erik, Sur les catégories spectrales localement distributives, C. R. Acad. Sci. Paris Sér. A-B 265 (1967), A14-A17.

[15] Roos, Jan-Erik, Sur la décomposition bornée des objets injectifs dans les catégories de Grothendieck, C. R. Acad. Sci. Paris Sér. A-B 266 (1968), A449-A452.

[16] Roos, Jan-Erik, Sur la structure des catégories spectrales et les coordonnées de von Neumann des treillis modulaires et complémentés, C. R. Acad. Sci. Paris Sér. A-B 265 (1967), A42-A45. 
[17] Roos, Jan-Erik, Sur la structure des catégories abéliennes localement noethériennes, C. R. Acad. Sci. Paris Sér. A-B 266 (1968), A701-A704.

[18] Roos, Jan-Erik, Sur l'anneau maximal de fractions des $A W^{*}$-algèbres et des anneaux de Baer, C. R. Acad. Sci. Paris Sér. A-B 266 (1968), A120-A123.

[19] Roos, Jan Erik, Locally Noetherian categories and generalized strictly linearly compact rings. Applications, in: Category Theory, Homology Theory and their Applications, II (Seattle, 1968), Springer, Berlin, 1969; pp. 197277.

[20] Roos, Jan-Erik, Coherence of general matrix rings and non-stable extensions of locally noetherian categories, ms. 1-15, and appendix 2 pages 1969.

[21] Roos, Jan-Erik, On the structure of abelian categories with generators and exact direct limits. Applications, 1969, ms. circa 300 pages (many of these results are published in CR-notes mentioned above).

[22] Palmér, Ingegerd; Roos, Jan-Erik, Formules explicites pour la dimension homologique des anneaux de matrices généralisées, C. R. Acad. Sci. Paris Sér. A-B 273 (1971), A1026-A1029.

[23] Roos, Jan-Erik, Détermination de la dimension homologique globale des algèbres de Weyl, C. R. Acad. Sci. Paris Sér. A-B 274 (1972), A23-A26.

[24] Roos, Jan-Erik, Proprietés homologiques des quotients primitifs des algèbres enveloppantes des algèbres de Lie semi-simples C. R. Acad. Sci. Paris Sér. A-B 276 (1973), A351-A354.

[25] Roos, Jan-Erik, Compléments à l'étude des quotients primitifs des algèbres enveloppantes des algèbres de Lie semi-simples, C. R. Acad. Sci. Paris Sér. A-B 276 (1973), A447-A450.

[26] Palmér, Ingegerd; Roos, Jan-Erik, Explicit formulae for the global homological dimensions of trivial extensions of rings, J. Algebra 27 (1973), 380-413.

[27] Roos, Jan-Erik, Sur l'algèbre Ext de Yoneda d'un anneau local de Golod, C. R. Acad. Sci. Paris Sér. A-B 286 (1978), A9-A12.

[28] Roos, Jan-Erik, Relations between Poincaré-Betti series of loop spaces and of local rings, in: Séminaire d'Algèbre Paul Dubreil, 31ème année (Paris, 1977-1978), Lecture Notes in Math. 740, Springer, Berlin, 1979; pp. 285322.

[29] Löfwall, Clas; Roos, Jan-Erik, Cohomologie des algèbres de Lie graduées et séries de Poincaré-Betti non rationnelles, C. R. Acad. Sci. Paris Sér. A-B 290 (1980), A733-A736.

[30] Roos, Jan-Erik, Homology of loop spaces and of local rings, in: 18th Scandinavian Congress of Mathematicians (Aarhus, 1980), Progr. Math. 11, Birkhäuser, Boston, Mass., 1981; pp. 441-468.

[31] Roos, Jan-Erik, Finiteness conditions in commutative algebra and solution of a problem of Vasconcelos, in: Commutative algebra (Durham, 1981), London Math. Soc. Lecture Note Ser. 72, Cambridge Univ. Press, Cambridge, 1982; pp. 179-203. 
[32] Roos, Jan-Erik, On the use of graded Lie algebras in the theory of local rings, in: Commutative algebra (Durham, 1981), London Math. Soc. Lecture Note Ser. 72, Cambridge Univ. Press, Cambridge, 1982; pp. 204-230.

[33] Roos, Jan-Erik (ed.), Algebra, algebraic topology and their interactions (Stockholm, 1983), Lecture Notes in Math. 1183, Springer, Berlin, 1986; see in particular his A mathematical introduction, pp. iii-viii.

[34] Backelin, Jörgen; Roos, Jan-Erik, When is the double Yoneda Ext-algebra of a local Noetherian ring again Noetherian?, in: Algebra, algebraic topology and their interactions (Stockholm, 1983), Lecture Notes in Math. 1183, Springer, Berlin, 1986; pp. 101-119.

[35] Roos, Jan-Erik, Homology of free loop spaces, cyclic homology and nonrational Poincaré-Betti series in commutative algebra, in: Algebra. Some current trends (Varna, 1986), Lecture Notes in Math. 1352, Springer, Berlin, 1988; pp. 173-189.

[36] Roos, Jan-Erik, Commutative non-Koszul algebras having a linear resolution of arbitrarily high order. Applications to torsion in loop space homology, C. R. Acad. Sci. Paris Sér. I Math. 316 (1993), 1123-1128.

[37] Roos, Jan-Erik, A computer-aided study of the graded Lie algebra of a local commutative Noetherian ring, J. Pure Appl. Algebra 91 (1994), 255-315.

[38] Roos, Jan-Erik, On the characterisation of Koszul algebras. Four counterexamples, C. R. Acad. Sci. Paris Sér. I Math. 321 (1995), 15-20.

[39] Roos, Jan-Erik, On computer-assisted research in homological algebra, in: Symbolic computation, new trends and developments (Lille, 1993), Math. Comput. Simulation 42 (1996), 475-490.

[40] Roos, Jan-Erik, A Description of the homological behaviour of families of quadratic forms in four variabless, in: Syzygies and Geometry (Boston, 1995), Northeastern Univ., Boston, MA, 1995; pp. 86-95.

[41] Roos, Jan-Erik, Koszul algebras and non-Koszul algebras, in: Syzygies and Geometry (Boston, 1995), Northeastern Univ., Boston, MA, 1995; pp. 9699.

[42] Löfwall, Clas; Roos, Jan-Erik, A nonnilpotent 1-2-presented graded Hopf algebra whose Hilbert series converges in the unit circle, Adv. Math. 130 (1997), 161-200.

[43] Roos, Jan-Erik; Sturmfels, Bernd, A toric ring with irrational Poincaré-Betti series, C. R. Acad. Sci. Paris Sér. I Math. 326 (1998), 141-146.

[44] Roos, Jan-Erik, Some non-Koszul algebras, in: Advances in geometry, Progr. Math., 172, Birkhäuser Boston, Boston, 1999; PP. 385-389.

[45] Fröberg, Ralf; Roos, Jan-Erik, An affine monomial curve with irrational Poincaré-Betti series, in: Commutative algebra, homological algebra and representation theory (Catania/Genoa/Rome, 1998), J. Pure Appl. Algebra 152 (2000), 89-92.

[46] Roos, Jan-Erik, Good and bad Koszul algebras, in preparation; cf. Abstracts Amer. Math. Soc. 19 (1998), 409. 
[47] Roos, Jan-Erik, Homological properties of quotients of exterior algebras, in preparation; cf. Abstracts Amer. Math. Soc., 21 (2000), 50-51.

[48] Roos, Jan-Erik, Modules with strange homological properties and Chebychev polynomials, to appear.

[49] Roos, Jan-Erik, A generalization of Golod homomorphisms of rings, in preparation.

This article may be accessed via WWW at http://www.rmi.acnet.ge/hha/ or by anonymous ftp at

ftp://ftp.rmi.acnet.ge/pub/hha/volumes/2002/n2a0/v4n2a0.(dvi,ps,pdf)

Luchezar L. Avramov avramov@math.unl.edu

Department of Mathematics,

University of Nebraska,

Lincoln, NE 68588,

USA 